\title{
Constrained Independence System and Triangulations of Planar Point Sets ${ }^{1}$
}

\author{
Siu-Wing Cheng ${ }^{2} \quad$ Yin-Feng $\mathrm{Xu}^{3}$
}

\begin{abstract}
We propose and study a new constrained independence system. We obtain a sequence of results, including a matching theorem for bases of the system and introducing a set of light elements which give a lower bound for the objective function of a minimization problem in the system. We then demonstrate that the set of triangulations of a planar point set can be modeled as constrained independence systems. The corresponding minimization problem in the system is the well-known minimum weight triangulation problem. Thus, we obtain two matching theorems for triangulations and a set of light edges (or light triangles) that give a lower bound for the minimum weight triangulation. We also prove directly a third matching theorem for triangulations. We show that the set of light edges is a superset of some subsets of edges of a minimum weight triangulation that were studied before.
\end{abstract}

\footnotetext{
${ }^{1}$ Research of the first author is supported partially by RGC grant HKUST 190/93E. Research of the second author is supported partially by RGC grants HKUST 190/93E and HKUST 181/93E.

${ }^{2}$ Department of Computer Science, The Hong Kong University of Science \& Technology, Clear Water Bay, Hong Kong. E-mail : scheng@cs.ust.hk

${ }^{3}$ School of Management, Xi'an Jiaotong University, Xi'an, Shaanxi, 710049, PRC. The work is done while the second author visits Department of Computer Science, The Hong Kong University of Science and Technology. E-mail : xuyinfeg@cs.ust.hk
} 


\section{Introduction}

Independence system plays a very important role in the study of discrete optimization problems and matroid is a well-known example [Wel76]. In this paper, we propose a constrained independence system and study some of its combinatorial properties. In particular, we prove a matching theorem for bases of such a system. We define a minimization problem for such a system and presents two heuristics that are based on a greedy approach and a local search method, respectively. We also study how to obtain a lower bound to the minimization problem.

The constrained independence problem can be used to model the set of all possible triangulations of a given planar point set. Thus, our result implies a matching between edges/triangles of different triangulations of the same point set. (The same matching theorem is also obtained independently by Aichholzer et al [AATR].) This is a new structural property of triangulations that were not known before. The minimization problem for a constrained independence system then translates to the open problem of finding a minimum weight triangulation. We present two lower bounds to this problem.

The definition of constrained independence system is very similar to that of matroid. However, the minimization for a constrained independence system can be shown to be NP-hard [Rot], while the optimization problem for matroid can be solved efficiently by a greedy strategy. This implies that if the minimum weight triangulation problem can be solved in polynomial time, then properties (perhaps geometrical) other than those captured by the constrained independence system have to be discovered.

The rest of this paper is organized as follows. In Section 2, we define the constrained independence system and prove several properties, including the matching theorem and a lower bound for the minimization problem. In Section 3, we model the triangulations with constrained independence systems. In Section 3.1, we first present two matching theorems for triangulations that follow from the result in Section 2. We also prove directly another different matching theorem for triangulations. In Section 3.2, we use the result in Section 2 to define light edges and light triangles, which will give lower bounds to the minimum weight triangulation problem. We also prove that the set of light edges is a superset of subsets of edges of minimum weight triangulations studied by Keil [Kei94] and Xu [Xu92].

\section{Constrained independence system}

Given $E$ a finite set of elements and $\mathcal{J}$ a nonempty collection of subsets of $E,(E, \mathcal{J})$ is an independence system if $Y \in \mathcal{J}$ whenever $X \in \mathcal{T}$ and $Y \subseteq X$. The elements of $\mathcal{J}$ are called independent sets. A maximal independent set is called a base. Any subset of $E$ that does not belong to $\mathcal{T}$ is 
called a dependent set. A minimal dependent set is called a circuit. A lot of combinatorial optimization problems can be formulated as optimization problems on different independence systems by associating appropriate non-negative weight $w(e)$ with each element $e$ of $E$,

We define a constrained independence system as follows. $(E, \mathcal{J})$ is a constrained independence system if it is an independence system and it satisfies the following conditions:

Condition(1). If $X, Y \in \mathcal{J}$ with $|Y|=p$ and $|X|=p+1$, then there is an element $e \in E-Y$ such that $Y \cup\{e\} \in \mathcal{J}$.

Condition(2). The cardinality of every circuit is two.

Fact. Every base of an independence system that satisfies condition(1) has the same cardinality.

The definition of constrained independence system is very similar to that of matroid. A matroid is an independence system such that if $X, Y \in \mathcal{J}$ with $|Y|=p$ and $|X|=p+1$, then there is an element $e \in X-Y$ such that $Y \cup\{e\} \in \mathcal{J}$. This is more restrictive than condition(1). Also, in general, a matroid may not satisfy condition(2).

\subsection{A matching theorem}

We prove a matching theorem for bases of a constrained independence system. Let $\mathcal{B}$ be the set of all bases.

Theorem 1 Given $B, B^{\prime} \in \mathcal{B}$, there exists a perfect matching between $B$ and $B^{\prime}$, such that if $e \in B$ and $e^{\prime} \in B^{\prime}$ are matched, then either $e=e^{\prime}$ or $\left\{e, e^{\prime}\right\}$ is a circuit.

Proof Construct a bipartite graph with vertex set $B$ and $B^{\prime}$. There is an edge between $e \in B$ and $e^{\prime} \in B^{\prime}$ if $e=e^{\prime}$ or $\left\{e, e^{\prime}\right\}$ is a circuit. For any subset $F \subseteq B$, let $N(F)$ be the set of vertices in $B^{\prime}$ that are adjacent to some vertex in $F$. We now show that $|N(F)| \geq|F|$ and thus, by the König-Hall matching theorem [Bol79], there is a perfect matching between $B$ and $B^{\prime}$.

We claim that $F \cup\left(B^{\prime}-N(F)\right) \in \mathcal{J}$. Otherwise, $F \cup\left(B^{\prime}-N(F)\right)$ contains some circuit $\left\{e, e^{\prime}\right\}$ (which contains exactly two elements by condition(2)) such that $e \in F$ and $e^{\prime} \in B^{\prime}-N(F)$. But this implies that $e^{\prime} \in N(F)$, a contradiction. Since $F \cup\left(B^{\prime}-N(F)\right) \in \mathcal{J}$ and all bases have the same cardinality, $\left|F \cup\left(B^{\prime}-N(F)\right)\right| \leq\left|B^{\prime}\right|$. Moreover, $\left|F \cup\left(B^{\prime}-N(F)\right)\right|=|F|+\left|B^{\prime}\right|-|N(F)|$. So we conclude that $|N(F)| \geq|F|$. This completes the proof. 


\subsection{The minimization problem}

In this section, we consider optimization problems for a constrained independence system which can be formulated as

$$
\min _{B \in \mathcal{B}} \sum_{e \in B} w(e)
$$

where $w(e)$ is the (non-negative) weight of $e$. In general, it is very difficult to find an optimal solution for the objective function. However, local optimum can be computed efficiently using a greedy approach.

A base $B$ is a local optimum if for every $e^{*} \in B$ and $e^{\prime} \in E-B$ such that $\left\{e^{\prime}\right\} \cup\left(B-\left\{e^{*}\right\}\right)$ is a base, we have $\sum_{e \in B} w(e) \leq \sum_{e \in\left\{e^{\prime}\right\} \cup\left(B-\left\{e^{*}\right\}\right)} w(e)$.

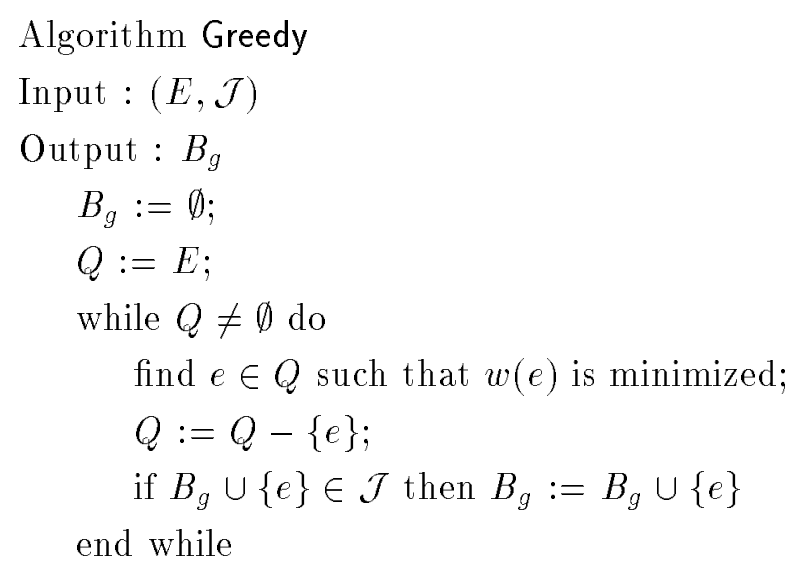

Theorem 2 The output of algorithm Greedy, $B_{g}$, is a base and is a local optimum.

Proof It is clear that $B_{g} \in \mathcal{J}$. Assume to the contrary that $B_{g}$ is not maximal. Then there exists $e \in E-B_{g}$ such that $B_{g} \cup\{e\} \in \mathcal{J}$, which implies that $\{e\} \cup A \in \mathcal{J}$ for all $A \subseteq B_{g}$. Thus, when $e$ was examined by algorithm Greedy, $e$ should have been included in $B_{g}$, a contradiction. Hence, $B_{g}$ is a base.

Assume to the contrary that $B_{g}$ is not a local optimum. Then there exists $e \in B_{g}$ and $e^{\prime} \in E-B_{g}$ such that $\left\{e^{\prime}\right\} \cup\left(B_{g}-\{e\}\right)$ is a base and $w\left(e^{\prime}\right)<w(e)$. Therefore, algorithm Greedy should examine $e^{\prime}$ before $e$. Since $\left\{e^{\prime}\right\} \cup\left(B_{g}-\{e\}\right) \in \mathcal{J},\left\{e^{\prime}\right\} \cup A \in \mathcal{J}$ for all $A \subseteq B_{g}-\{e\}$. Thus, $e^{\prime}$ will be included and so $e$ will not be included later, which contradicts our assumption.

Remark. Since the proof of Theorem 2 does not make use of condition(1) and condition(2), the result is true for all independence systems. 
The greedy solution can be viewed as an approximate solution to the minimization problem. Sometimes, we may be given a base $B$ and we are asked to obtain an improved solution. We can handle this problem with algorithm ESA which uses repeated element substitution to obtain an improved solution. A base $B^{\prime}$ is obtained from another base $B$ by element substitution, if there exists $e \in B$ and $\epsilon^{\prime} \in B^{\prime}-B$ such that $B^{\prime}=\left\{\epsilon^{\prime}\right\} \cup(B-\{e\})$.

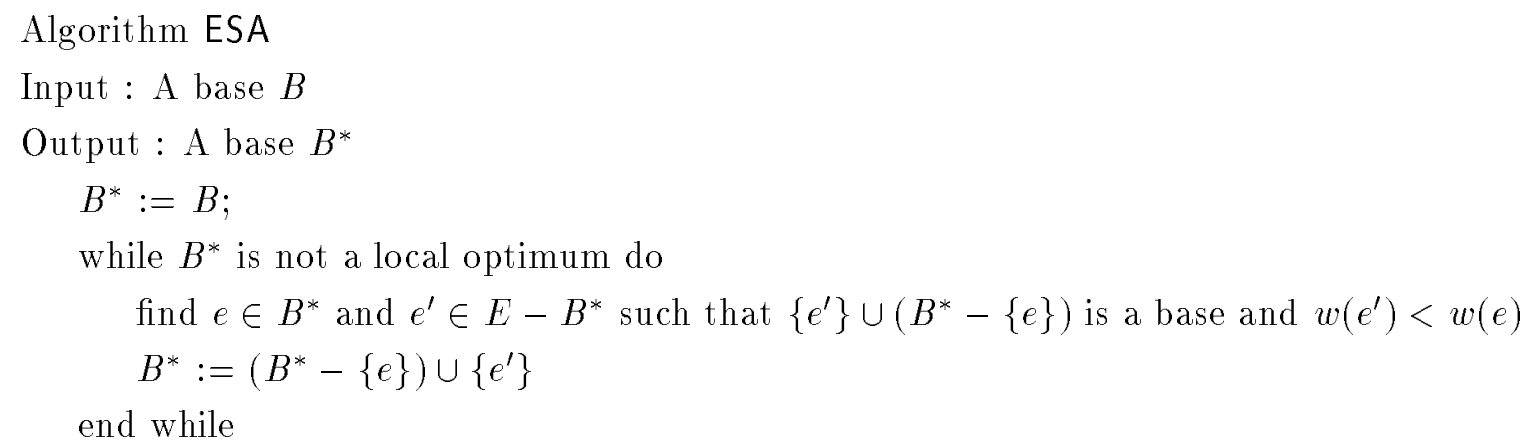

From the working of algorithm ESA, it is clear that the following is true.

Theorem 3 Let $B$ and $B^{*}$ be the input and output of algorithm ESA, respectively. Then $B^{*}$ is a local optimum and $\sum_{e \in B^{*}} w(e) \leq \sum_{e \in B} w(e)$. If $B$ is not a local optimum, then the inequality holds strictly.

We do not know of any polynomial-time algorithm that can solve the general minimization problem. Thus, it may be helpful to derive lower bound to the objective function, which can then guide the running of some heuristics or exhaustive search algorithms. We describe one such lower bound in the following. An element $e \in E$ is light if $w(e)<w\left(e^{\prime}\right)$ for all $e^{\prime} \in E$ such that $\left\{e, e^{\prime}\right\} \notin \mathcal{J}$. Let $L(E)$ be the set of all light elements.

Theorem $4 L(E) \in \mathcal{J}$ and $\sum_{e \in L(E)} w(e) \leq \min _{B \in \mathcal{B}} \sum_{e \in B} w(e)$.

Proof Assume to the contrary that $L(E) \notin \mathcal{J}$. Then there exists a circuit in $L(E)$. By condition(2), this circuit is $\left\{e_{1}, e_{2}\right\} \notin \mathcal{J}$ for some $e_{1}, e_{2} \in L(E)$. But this implies that $w\left(e_{1}\right)<w\left(e_{2}\right)$ and $w\left(e_{2}\right)<w\left(e_{1}\right)$, a contradiction. Let $B^{*}$ be a base such that $\sum_{e \in B^{*}} w(e)=\min _{B \in \mathcal{B}} \sum_{e \in B} w(e)$. Since $L(E) \in \mathcal{J}$, by condition(1), there is a base $B$ such that $L(E) \subseteq B$. We apply Theorem 1 to $B$ and $B^{*}$. Thus, every $e_{1} \in L(E)$ is matched with an $e_{2} \in B^{*}$ such that either $e_{1}=e_{2}$ or $\left\{e_{1}, e_{2}\right\} \notin \mathcal{J}$. If $\left\{e_{1}, e_{2}\right\} \notin \mathcal{J}$, then $w\left(e_{1}\right)<w\left(e_{2}\right)$ as $e_{1} \in L(E)$. So we conclude that $w\left(e_{1}\right) \leq w\left(e_{2}\right)$ and hence $\sum_{e \in L(E)} w(e) \leq \sum_{e \in B^{*}} w(e)$. 
So $\sum_{e \in L(E)} w(e)$ is a lower bound to $\min _{B \in \mathcal{B}} \sum_{e \in B} w(e)$. In the proof above, we mention that there exists a base $B$ such that $L(E) \subseteq B$. We can identify one such possible $B$ to be $B_{g}$, where $B_{g}$ is the output of algorithm Greedy.

Theorem $5 L(E) \subseteq B_{g}$.

Proof Let $e \in L(E)$. For all $e^{\prime} \in E$, if $\left\{e, e^{\prime}\right\}$ is a circuit, then $w(e)<w\left(e^{\prime}\right)$ as $e \in L(E)$. Therefore, no such $e^{\prime}$ can be examined before $e$ in algorithm Greedy, which implies that $e$ should be included in the greedy solution when it is examined. This completes the proof.

\section{Applications in triangulations}

Let $S$ be a finite planar point set. To simplify our exposition, we assume that no three points in $S$ are collinear. Let $E(S)$ denote the set of edges with endpoints in $S$. Let $\Delta(S)$ denote the set of empty triangles with vertices in $S$. (In this paper, we treat a triangle as the closed region bounded by its boundary edges.) A triangle is empty if its interior does not contain any point in $S$. Given an edge $e$ (resp. a triangle $\Delta$ ), we use inte (resp. int $\Delta$ ) to denote the interior of $e$ (resp. $\Delta$ ).

A triangulation $T_{e}(S)$ is a maximal subset of $E(S)$ such that inte $e_{1}$ ninte $e_{2}=\emptyset$ for any two distinct edges $e_{1}, e_{2} \in T_{e}(S)$. Let $n$ denote the cardinality of $S$. From Euler's formula, we have [Ede87]

$$
\left|T_{e}(S)\right|=3 n-3-|C H(S)|,
$$

where $C H(S)$ denotes the set of boundary edges of the convex hull of $S$. We can equivalently view a triangulation as a maximal subset $T_{t}(S)$ of $\Delta(S)$ such that int $\Delta_{1} \cap$ int $\Delta_{2}=\emptyset$ for any two distinct triangles $\Delta_{1}, \Delta_{2} \in T_{t}(S)$. The following can also derived from Euler's formula.

$$
\left|T_{t}(S)\right|=2 n-2-|C H(S)| .
$$

\subsection{Matching theorems for triangulations}

Given a point set $S$ of size $n$, define $\mathcal{J}_{e}(S)$ to be the collection of subsets of $E(S)$ such that for all $X \in \mathcal{J}_{e}(S)$ and $e_{1}, e_{2} \in X$, inte $e_{1} \cap$ inte $e_{2}=\emptyset$. We claim that $\left(E(S), \mathcal{J}_{e}(S)\right)$ is a constrained independence system. By Equation 1, every base in $\mathcal{J}_{e}(S)$ has the same cardinality and hence condition(1) is satisfied. Condition(2) is also satisfied since the smallest dependent set must be an intersecting pair of edges in $E(S)$. By Theorem 1, we have the following corollary.

Corollary 1 Given a finite point set $S$ and two triangulations $T_{1}, T_{2} \in \mathcal{J}_{e}(S)$, there exists a perfect matching between $T_{1}$ and $T_{2}$ such that if $e_{1} \in T_{1}$ and $e_{2} \in T_{2}$ are matched, then int $e_{1} \cap$ int $e_{2} \neq \emptyset$. 

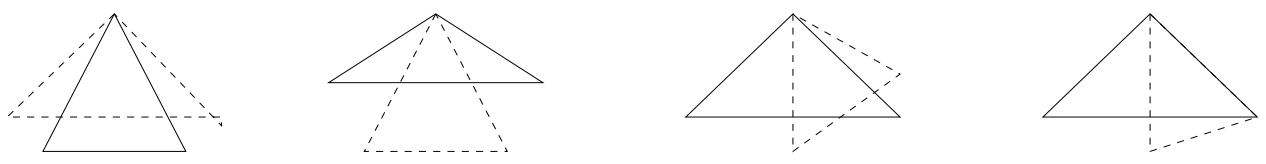

Figure 1:

We can also consider the constrained independence system $\left(\Delta(S), \mathcal{J}_{t}(S)\right)$, where $\mathcal{J}_{t}(S)$ is the collection of subsets of $\Delta(S)$ such that for all $X \in \mathcal{J}_{t}(S)$ and $\Delta_{1}, \Delta_{2} \in X$, int $\Delta_{1} \cap \operatorname{int} \Delta_{2}=\emptyset$. We then obtain another corollary from Theorem 1.

Corollary 2 Given a finite point set $S$ and two triangulations $T_{1}, T_{2} \in \mathcal{J}_{t}(S)$, there exists a perfect matching between $T_{1}$ and $T_{2}$ such that if $\Delta_{1} \in T_{1}$ and $\Delta_{2} \in T_{2}$ are matched, then int $\Delta_{1} \cap$ int $\Delta_{2} \neq$ $\emptyset$.

Corollaries 1 and 2 are also obtained independently by [AATR]. We can actually obtain a matching theorem stronger than Corollary 2 by applying the König-Hall Theorem directly.

Theorem 6 Given a finite point set $S$ and two triangulations $T_{1}, T_{2} \in \mathcal{J}_{t}(S)$, there exists a perfect matching between $T_{1}$ and $T_{2}$ such that if $\Delta_{1} \in T_{1}$ and $\Delta_{2} \in T_{2}$ are matched, then

1. $\operatorname{int} \Delta_{1} \cap \operatorname{int} \Delta_{2} \neq \emptyset$

2. $V\left(\Delta_{1}\right) \cap V\left(\Delta_{2}\right) \neq \emptyset$, where $V\left(\Delta_{i}\right)$ denotes the vertex set of $\Delta_{i}, i=1$ to 2 .

Proof Construct a bipartite graph with one vertex set corresponding to triangles in $T_{1}$ and the other vertex set corresponding to triangles in $T_{2}$. For convenience, we use $\Delta_{i} \in T_{i}, i=1$ or 2 , to denote both a triangle and the corresponding vertex in the bipartite graph. There is an edge between $\Delta_{1} \in T_{1}$ and $\Delta_{2} \in T_{2}$ if int $\Delta_{1}$ nint $\Delta_{2} \neq \emptyset$ and $V\left(\Delta_{1}\right) \cap V\left(\Delta_{2}\right) \neq \emptyset$. For any subset $F \subseteq T_{1}$, let $N(F) \subseteq T_{2}$ be the set of triangles that are neighbors of some triangle in $F$. To complete the proof, it suffices to show that $|N(F)| \geq|F|$. Let $x$ be a vertex of a triangle $\Delta_{1}$ in $F$. There is a set of triangles in $N(F)$ that share the vertex $x$ with $\Delta_{1}$. Moreover, the union of the angles of these triangles at $x$ must contain the angle of $\Delta_{1}$ at $x$. Thus, if we sum up the angles of all triangles in $N(F)$ and $F$, we obtain the inequality $\pi|N(F)| \geq \pi|F|$, which implies that $|N(F)| \geq|F|$.

By Theorem 6, two matched triangles are either identical or they are in one of the four configurations shown in Figure 1.

\subsection{Light edges and triangles}

Given a finite point set $S$ in the plane, the minimum weight triangulation problem is to triangulate the point set so that the sum of edge lengths is minimized. The complexity of the problem has 
not been resolved: neither is it known to be NP-hard nor is a polynomial-time algorithm known to exist [GJ79]. Also, no approximation algorithm is known that achieves a constant approximation ratio [HP90]. The best approximation algorithm is due to Plaisted and Hong [PH87] which achieves an approximation ratio of $O(\log n)$, where $n$ is the size of the given point set. In this section, we make use of Theorem 4 to give a lower bound on the sum of edge lengths in a minimum weight triangulation.

Given an edge $e \in E(S)$, define $w(e)$ to be its length. An edge $e$ is light if $w\left(e^{\prime}\right)>w(e)$ for all edge $e^{\prime} \in L(S)$ such that inte $\cap$ int $e^{\prime} \neq \emptyset$. We use $L(E(S))$ to denote the set of light edges.

We can define light triangles similarly. Given a triangle $\Delta \in \Delta(S)$, define $w(\Delta)$ to be its perimeter. A triangle $\Delta$ is light if $w\left(\Delta^{\prime}\right)>w(\Delta)$ for all triangle $\Delta^{\prime} \in \Delta(S)$ such that int $\Delta \cap$ int $\Delta^{\prime} \neq$ $\emptyset$. We use $L(\Delta(S))$ to denote the set of light triangles.

In [KR85], a $\beta$-skeleton of a planar point set is introduced and it is proved in [Kei94] that a $\sqrt{2}$-skeleton is a subgraph of a minimum weight triangulation. We describe the definition of $\beta$ skeleton below. For $\beta>1$, the forbidden neighborhood for points $x$ and $y$ is defined to be the union of the two disks of radius $\beta \cdot d(x, y) / 2$ that pass through both $x$ and $y$ (see Figure 2 ), where $d(x, y)$ denotes the distance between $x$ and $y$. The edge $x y$ is in the $\beta$-skeleton if the forbidden neighborhood for $x$ and $y$ does not contain any point of the given point set. (There is actually another variant of $\beta$-skeleton, the definition of which is based on lune-like neighborhood provided in [KR85]. The above results refer to the disk-based forbidden neighborhood described.) We use $\beta(E(S))$ to denote the $\beta$-skeleton, for $\beta>1$.

We now prove that $\beta(E(S)) \subseteq L(E(S))$ for $\beta \geq 2 / \sqrt{3}$ which implies that the $\sqrt{2}$-skeleton is a subset of $L(E(S))$.

Theorem $7 \beta(E(S)) \subseteq L(E(S))$ for $\beta \geq 2 / \sqrt{3}$.

Proof Refer to Figure 2. Let the union of the two disks shown be the forbidden neighborhood of $x$ and $y$. Suppose that the forbidden neighborhood is empty. Note that the disk centers $v$ and $w$ are on opposite sides of $x y$. It suffices to show that every edge that intersects $x y$ is longer than $x y$.

Suppose that an edge $a b$ intersects $x y$ at a point $z$ and the disk centers are on opposite sides of $a b$. By geometry, $a z$ is longer than $y z$ and $b z$ is longer than $x z$. Thus $a b$ is longer than $x y$.

Suppose that an edge $b c$ intersects $x y$ and $v$ and $w$ lie on the same side of $b c$. Draw a line segment from $c$ through $x$ to a point $d$ on the boundary of the other disk. By geometry, $b c$ is longer than $c d$. Thus, it suffices to show that $c d$ is at least as long as $x y$ for $\beta \geq 2 / \sqrt{3}$. Imagine that $c d$ is actually an elastic rod that passes through $x$ and connects two points on the boundary of the two disks. If we move the endpoints of the elastic rod to rotate it about $x$, its length will change. 


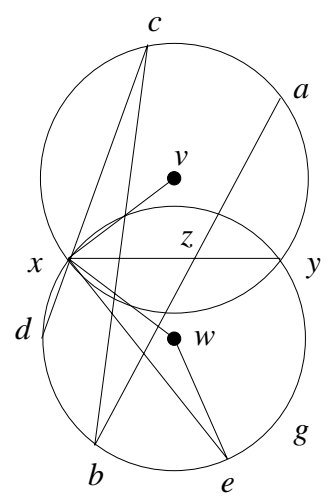

Figure 2:

The length of $c x$ is a convex function in Lcxy. Similarly, the length of $d x$ is a convex function in $L d x y$. Since length of $c d$ is the sum of the lengths of $c x$ and $d x$, it is also a convex function in $L c x y$. Moreover, length of $c x$ equals length of $d x$ when $c d$ is perpendicular to $x y$. Therefore, the length of $c d$ is maximized when $c d$ is perpendicular to $x y$.

Thus, the length of $c d$ achieves the minimum when $c$ coincides with $x$ and $d$ moves to a point $e$ such that $e x$ is a tangent to the disk with center $v$. Refer to Figure 2. Let $r$ be the radius of the two disks. Let $\theta=\angle v x y=\angle w x y$. Then in order that $x y$ is light, we require that $2 r \cos (\pi / 2-2 \theta) \geq$ $d(x, y)$. Since $d(x, y) / 2 r=\cos \theta$, this is equivalent to $\sin 2 \theta \geq \cos \theta$ or $\cos \theta \leq \sqrt{3} / 2$. Since $\beta=2 r / d(x, y)=1 / \cos \theta$, this is equivalent to requiring that $\beta \geq 2 / \sqrt{3}$.

Another kind of subgraph of a minimum triangulation is the intersection of the edge set of all triangulations. This common intersection is called the stable line segments in [Xu92]. Since each stable line segment is not intersected by any other edge in $E(S), L(E(S))$ contains all the stable line segments by definition.

By considering the constrained independence systems $\left(E(S), \mathcal{J}_{e}(S)\right)$ and $\left(\Delta(S), \mathcal{J}_{t}(S)\right)$ and applying Theorem 4, we obtain the following lower bounds for a minimum weight triangulation.

Corollary 3 Let $M W T_{e}(S)$ and $M W T_{t}(S)$ be the set of edges and triangles, respectively, of a minimum weight triangulation.

1. $\sum_{e \in L(E(S))} w(e) \leq \sum_{e \in M W T_{e}(S)} w(e)$

2. $\sum_{\Delta \in L(\Delta(S))} w(\Delta) \leq \sum_{\Delta \in M W T_{t}(S)} w(\Delta)$.

We want to point out that the two formulations $\left(E(S), \mathcal{J}_{e}(S)\right)$ and $\left(E(S), \mathcal{J}_{t}(S)\right)$ are different. Refer to Figure 3(a). By construction, $a c$ is longer than $b d$, $a d$ and $b c$ are longer than $b d$, and $a b$ and $b c$ are the shortest edges. So the set of light edges $\{a b, b c, b d, a d, c d\}$ is exactly the minimum 


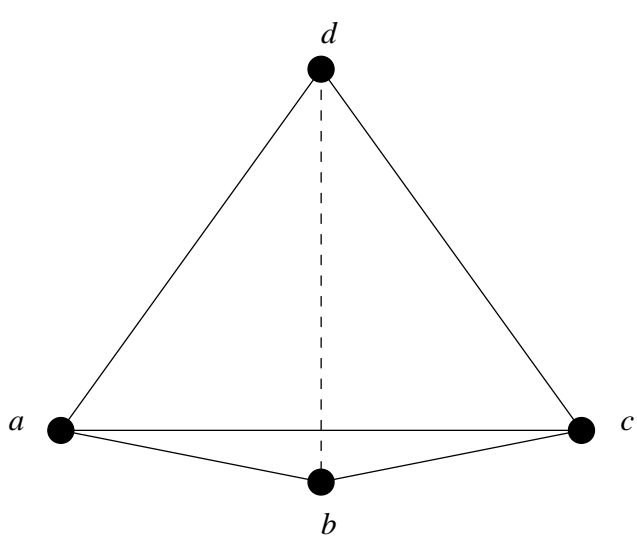

(a)

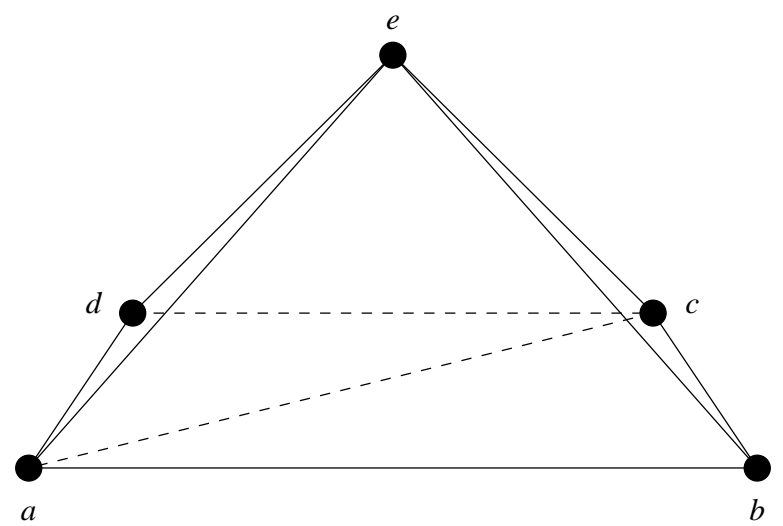

(b)

Figure 3:

weight triangulation. The triangle $a b c$ has the smallest perimeter and it is the only light triangle. Thus, in this example, running Greedy on edges will give us a better triangulation (the optimal one) than running Greedy on triangles. Refer to Figure 3(b). By construction, $a d$ and $b c$ are shortest and $c d$ is shorter than ae and be. Also, the sum of lengths of ae and be is less than the sum of lengths of $c d$ and $a c$. So the set of light edges is $\{a d, a b, b c, c d, d e, c e\}$. The set of light triangles is $\{a d e, b c e\}$. Thus, in this example, running Greedy on triangles will give us a better triangulation (the optimal one) than running Greedy on edges.

Theorem 5 implies that $L(E(S))$ is a subset of the greedy triangulation. Given any triangulation, we can use algorithm ESA to try to obtain an improved triangulation. The repeated element substitution in algorithm ESA translates to repeated swapping of diagonals of convex quadrilaterals formed by two neighboring triangles.

\section{Conclusion and related work}

We introduce the constrained independence system and prove some properties which lead to some structural characterization about triangulations of a planar point set. Whether there exists stronger matching theorems for constrained independence system or triangulations is open. It is also interesting to study the application of the matching theorem to the minimum weight triangulation problem. By considering triangulations directly, Aichholzer et al [AATR] independently obtained Corollary 1 and Corollary 2. They can also construct a perfect matching between edges in an arbitrary triangulation and edges in $E(S)$, such that the length sum of matched edges in $E(S)$ is minimized. This provides a lower bound to the minimum weight triangulation problem. If the greedy triangulation is used to construct such a matching, then the lower bound derived is an 
improvement of the length sum of light edges.

\section{Acknowledgement}

We would like to thank Herbert Edelsbrunner and Mordecai Golin for discussion about this work. We also thank Günter Rote for helpful discussion and carefully reading a draft of this paper.

\section{References}

[AATR] O. Aichholzer, F. Aurenhammer, M. Taschwer, and G. Rote. Manuscript, 1994.

[Bol79] B. Boldobás, Graph Theory. An Introductory Course, Springer-Verlag, 1979.

[Ede87] H. Edelsbrunner, Algorithms in Combinatorial Geometry, Springer-Verlag, 1987.

[GJ79] M. Garey and D. Johnson, Computers and Intractability. A guide to the Theory of NP-completeness, Freeman, 1979.

[HP90] L. HeAth and S. PEMmaraju, New results for the minimum weight triangulation problem, Tech. Report TR 90-65, Virginia Polytechnic Institute and State University, 1990.

[Kei94] M. KeIL, Computing a subgraph of the minimum weight triangulation, Computational Geometry: Theory and Applications, 4 (1994), pp. 13-26.

[KR85] D. Kirkpatrick and J. Radke, A framework for computational morphology, in Computational Geometry, G. Toussaint, ed., Elsevier, Amsterdam, 1985, pp. 217-248.

[PH87] D. Plaisted And J. Hong, A heuristic triangulation algorithm, Journal of Algorithms, 8 (1987), pp. 405-437.

[Rot] G. Rote. personal communication.

[Wel76] D. Welsh, Matroid Theory, Academic Press, New York, 1976.

[Xu92] Y. Xu, Minimum weight triangulation problem of a planar point set, $\mathrm{PhD}$ thesis, Institute of Applied Mathematics, Academia Sinica, Beijing, 1992. 